\title{
Three-Dimensional Orthogonal Vector Basis Functions for Time-Domain Finite Element Solution of Vector Wave Equations
}

\author{
Dan Jiao, Member, IEEE, and Jian-Ming Jin, Fellow, IEEE
}

\begin{abstract}
Three-dimensional (3-D) orthogonal vector basis functions are developed for the time-domain finite element solution of vector wave equations. These basis functions enforce both the tangential continuity of the electric field and the normal continuity of the electric flux. The stability of the resulting time-domain finite element schemes is investigated and demonstrated to be guaranteed. The use of the proposed basis functions completely eliminates the matrix solution at each time step required by the time-domain finite element solution of vector wave equations. The computational cost thereby scales as $\mathcal{O}\left(N_{t} N\right)$ with $N_{t}$ and $N$ denoting the number of time steps and the number of unknowns, respectively. Defined over tetrahedral elements, the proposed basis functions increase the solution efficiency without compromising the geometry modeling flexibility. Both numerical results and comparison with traditional vector basis functions demonstrate the accuracy as well as the efficiency of the proposed three-dimensional orthogonal vector bases.
\end{abstract}

Index Terms-Electromagnetic scattering, electromagnetic transient analysis, finite-element methods, numerical analysis.

\section{INTRODUCTION}

B ECAUSE of their potential to generate wide-band data and model nonlinear materials, numerical schemes for simulating electromagnetic transients have grown increasingly popular in recent years. These numerical schemes can be categorized into two classes. Schemes in the first class solve time-domain partial differential equations [1], [2]. Schemes in the second class tackle time-domain integral equations (TDIE) [3]-[5]. Compared to the first class, TDIE-based schemes have certain advantages when analyzing scattering or radiation by impenetrable or homogeneous objects that reside in a homogeneous and unbounded medium. The reasons are twofold: First, they limit the unknowns on the surface of the object, and second, they satisfy the radiation condition naturally via the Green's function. However, when the numerical simulation involves complicated inhomogeneity, the partial differential equation-based solvers often prove to be more convenient and efficient. Especially, the finite-difference time-domain (FDTD) method [1] turns out to be the most popular tool for transient analysis involving complex inhomogeneous bodies. However, this method suffers from the well-known staircase problem,

Manuscript received January 31, 2001; revised December 18, 2001. This work was supported by a grant from AFOSR via the MURI Program under Contract F49620-96-1-0025.

The authors are with the Center for Computational Electromagnetics, Department of Electrical and Computer Engineering, University of Illinois at Urbana-Champaign Urbana, IL 61801 USA.

Digital Object Identifier 10.1109/TAP.2003.808524 and its removal requires much more effort in the sacrifice of computational resources. In contrast, the time-domain finite element method (TDFEM) can easily handle both complex geometry and inhomogeneous media, which cannot be achieved by either the FDTD- or the TDIE-based schemes.

During the past few years, a variety of TDFEM schemes have been proposed [2], [6]-[19]. These schemes fall into two categories. One directly discretizes Maxwell's equations [6]-[9], [18], which typically results in an explicit, finite difference-like leap-frog scheme that does not leverage our extensive knowledge of frequency-domain FE solvers. The other discretizes the second-order vector wave equation, also known as the curl-curl equation, obtained by eliminating one of the field variables from Maxwell's equations [10]-[16], [19]. Despite its ability to handle unstructured meshes and its capacity to impose continuity conditions across material interfaces, the TDFEM does not enjoy widespread popularity compared to the FDTD method. There are two major reasons. First, in contrast to the FDTD for which numerous absorbing boundary conditions (ABCs) have been extensively developed and investigated, research on accurate $\mathrm{ABCs}$ for the TDFEM is very scant: for a long time only first- and second-order $\mathrm{ABCs}$ have been implemented [17]-[19]. Second, unlike the FDTD method, the TDFEM algorithms that are based on the second-order vector wave equation require the solution of a matrix equation in each time step. The first situation is changing rapidly. The recently developed time-domain finite element-boundary integral method [20], [21] provides a powerful numerical technique for solving open-region electromagnetic scattering and radiation problems in the time domain. This method adopts the boundary integral representation to accurately truncate the computational domain and the multilevel plane wave time domain algorithm to efficiently evaluate the boundary integrals. The second issue, however, has yet to be resolved satisfactorily. Several lumping techniques [10], [16] have been proposed to render the mass matrix diagonal to obviate the need for its inversion. Unfortunately, these lumping procedures often introduce significant errors in the TDFEM solution and, for unstructured meshes, they are likely to produce zero or negative diagonal elements, which result in the definite instability [2]. A recently developed approach [15] avoids lumping altogether by constructing a set of orthogonal vector basis functions that yield a diagonal mass matrix. However, only two-dimensional (2-D) orthogonal vector basis functions have been constructed so far.

This paper presents a set of three-dimensional (3-D) orthogonal vector basis functions for the TDFEM solution of vector 
wave equations. The expressions of the proposed basis functions are described in Section II. Both the completeness and orthogonality properties are analyzed. Section III presents the orthogonal TDFEM solution based on the proposed basis functions. The stability of the TDFEM using these orthogonal bases is examined in Section IV. Section V demonstrates the efficiency and accuracy of the TDFEM using the orthogonal bases by numerical results and comparison with the TDFEM using traditional vector basis functions.

\section{ORTHOGONAL VECTOR BASIS FUNCTIONS}

To use the FEM, the computational domain is first subdivided into small tetrahedral elements. The electric flux within each element is then expanded as

$$
\mathbf{D}(\mathbf{r}, t)=\sum_{i=1}^{n} u_{i}(t) \mathbf{N}_{i}^{e}
$$

where $n$ denotes the number of basis functions per element, and $\mathbf{N}_{i}^{e}$ and $u_{i}$ denote the vector expansion functions and corresponding expansion coefficients, respectively. To obviate the need for a matrix solution in each time step, $\mathbf{N}_{i}^{e}$ must be orthogonal:

$$
\left\langle\mathbf{N}_{i}^{e}, \mathbf{N}_{j}^{e}\right\rangle=\iiint_{V^{e}} \mathbf{N}_{i}^{e} \cdot \mathbf{N}_{j}^{e} d V=\delta_{i j}
$$

where $V^{e}$ denotes the volume of element $e$ and $\delta_{i j}$ is the Kronecker delta function. Using numerical integration, (2) can be expressed as

$$
\left\langle\mathbf{N}_{i}^{e}, \mathbf{N}_{j}^{e}\right\rangle=\sum_{\ell=1}^{4} \alpha_{\ell} \mathbf{N}_{i}^{e}\left(m_{\ell}\right) \cdot \mathbf{N}_{j}^{e}\left(m_{\ell}\right)=\delta_{i j}
$$

where $m_{\ell}$ denotes the central point of facet $\ell$ of element $e$, and the coefficients $\alpha_{\ell}$ are chosen such that the numerical integration is at least second-order accurate.

The most widely used vector basis functions for the correct representation of electric and magnetic fluxes are the Whitney 2 -forms, also known as the facet elements, given by

$$
\mathbf{U}_{i}=2\left(\xi_{j} \nabla \xi_{k} \times \nabla \xi_{m}+\xi_{k} \nabla \xi_{m} \times \nabla \xi_{j}+\xi_{m} \nabla \xi_{j} \times \nabla \xi_{k}\right)
$$

for facet $i$ that connects nodes $j, k$, and $m$, where $\xi_{j}, \xi_{k}$, and $\xi_{m}$ represent the normalized volume coordinates. It is well known that the normal component of $\mathbf{U}_{i}$ is continuous across element boundaries, and its flux is one through facet $i$ and zero through all other facets. Hence, $\mathbf{U}_{i}$ guarantees the normal continuity of the fluxes across the element interfaces, while allowing for a tangential discontinuity. Therefore, they can represent the electric and magnetic fluxes correctly. However, these functions do not satisfy the orthogonality defined in (3). To make them orthogonal, we first construct a set of $\mathbf{P}$ basis functions which are derived as

$$
\begin{aligned}
\mathbf{P}_{i}= & N_{\mathbf{P}_{i}}\left\{\mathbf{U}_{i}-18\left(\xi_{j} \xi_{k} \xi_{m}+\xi_{i} \xi_{k} \xi_{m}+\xi_{i} \xi_{j} \xi_{k}\right) \nabla \xi_{m} \times \nabla \xi_{j}\right. \\
& -18\left(\xi_{j} \xi_{k} \xi_{m}+\xi_{i} \xi_{j} \xi_{m}+\xi_{i} \xi_{j} \xi_{k}\right) \nabla \xi_{k} \times \nabla \xi_{m} \\
& -18\left(\xi_{j} \xi_{k} \xi_{m}+\xi_{i} \xi_{k} \xi_{m}+\xi_{i} \xi_{j} \xi_{m}\right) \nabla \xi_{j} \times \nabla \xi_{k} \\
& \left.+54 \xi_{j} \xi_{k} \xi_{m}\left|\nabla \xi_{i}\right|^{-2}\left[\nabla \xi_{i} \cdot\left(\nabla \xi_{j} \times \nabla \xi_{k}\right)\right] \nabla \xi_{i}\right\}
\end{aligned}
$$

in which $N_{\mathbf{P}_{i}}$ denotes the normalization coefficient given by

$$
N_{\mathbf{P}_{i}}=\left|\nabla \xi_{i}\right|\left|\mathbf{U}_{i}\left(m_{i}\right) \cdot \nabla \xi_{i}\right|^{-1} .
$$

The normalization coefficient is chosen to ensure that the component of $\mathbf{P}_{i}\left(m_{i}\right)$ along the normal direction of facet $i$ is unity. Despite the complex form of (5), it can readily be proven that $\mathbf{P}$ basis preserves the property of Whitney 2 -form, that is, the normal continuity of the flux across the element boundaries. Specifically, its flux is $N_{\mathbf{P}_{i}}$ through facet $i$ and zero through all other facets. Furthermore, it can be observed that $\mathbf{P}_{i}$ is purely normal at the central point of facet $i$ and is zero at the central points of the other facets. Hence, $\mathbf{P}$ basis constitutes an orthogonal set based on the orthogonality defined in (3). However, by subtracting from $\mathbf{U}$ the four terms as shown in (5), the basis function $\mathbf{P}$ destroys the completeness of the original Whitney facet elements. This can be seen easily from the vanished tangential components at the central point of each facet. To remedy this problem, we construct another two sets of basis functions $\mathbf{Z}$ and $\mathbf{T}$ :

$$
\begin{aligned}
& \mathbf{Z}_{i}=N_{\mathbf{Z}_{i}} \epsilon_{r} \xi_{j} \xi_{k} \xi_{m} \hat{t}_{i} \\
& \mathbf{T}_{i}=N_{\mathbf{T}_{i}} \epsilon_{r} \xi_{j} \xi_{k} \xi_{m} \hat{n}_{i} \times \hat{t}_{i}
\end{aligned}
$$

in which $\hat{t}_{i}$ and $\hat{n}_{i}$ denote the unit vectors tangential and normal to facet $i$, respectively. The $N_{\mathbf{Z}_{i}}$ and $N_{\mathbf{T}_{i}}$ are normalization coefficients given by

$$
N_{\mathbf{Z}_{i}}=N_{\mathbf{T}_{i}}=27
$$

which make the projection of $\epsilon_{r}{ }^{-1} \mathbf{Z}_{i}\left(m_{i}\right)$ along $\hat{t}_{i}$ as well as $\epsilon_{r}{ }^{-1} \mathbf{T}_{i}\left(m_{i}\right)$ along $\hat{n}_{i} \times \hat{t}_{i}$ to be unity. The introduction of relative permittivity $\epsilon_{r}$ in (7) permits the discontinuity of the tangential fluxes across the element interfaces. Otherwise, two sets of degrees of freedom must be assigned across the element boundary, which would increase the number of unknowns. Clearly, both $\mathbf{Z}$ and $\mathbf{T}$ are orthogonal by themselves and are mutually orthogonal with each other. In addition, they are orthogonal with $\mathbf{P}$ basis functions. Interestingly, the subtracted terms in basis function $\mathbf{P}$ from Whitney facet elements, which make $\mathbf{P}$ incomplete, are compensated by bases $\mathbf{Z}$ and $\mathbf{T}$. As a consequence, the vector basis functions $\mathbf{P}, \mathbf{Z}$, and $\mathbf{T}$ constitute an orthogonal as well as a complete set of bases to represent the electric and magnetic fluxes. The vector plots of these bases are shown in Fig. 1. Worth to be mentioned is that compared to the zeroth-order edge elements, the orthogonal vector bases involve more degrees of freedom. However, the accuracy is increased at the same time because the expansion (1) enforces both the tangential continuity of the electric field intensity and the normal continuity of the electric flux density explicitly at the central point (interpolation point) of each element facet, whereas the edge elements enforce only the tangential continuity explicitly. We should also note that although the proposed orthogonal vector bases have higher-order terms, they are only complete to the zeroth order and the integration formula in (3) only evaluates the terms up to the second-order accurately and further exploration is needed to understand the effect of higher-order terms on the accuracy of the solution. 


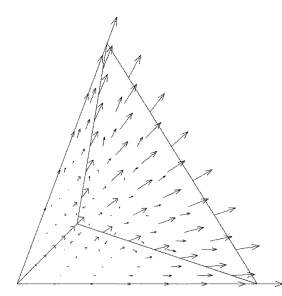

(a)

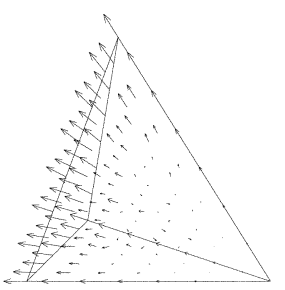

(b)

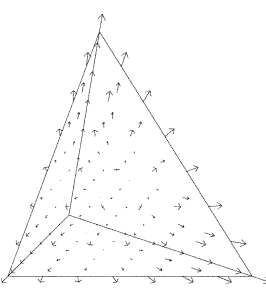

(c)

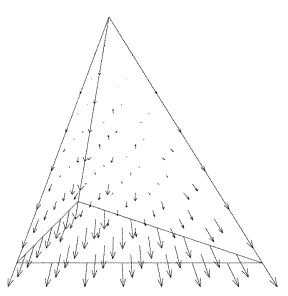

(d)

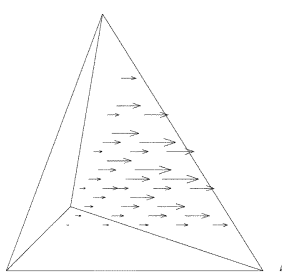

(e)

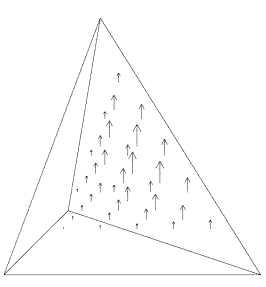

(f)

Fig. 1. Vector plots of 3-D orthogonal vector basis functions. (a) $\mathbf{P}_{1}$. (b) $\mathbf{P}_{2}$. (c) $\mathbf{P}_{3}$. (d) $\mathbf{P}_{4}$. (e) $\mathbf{Z}_{1}$. (f) $\mathbf{T}_{1}$.

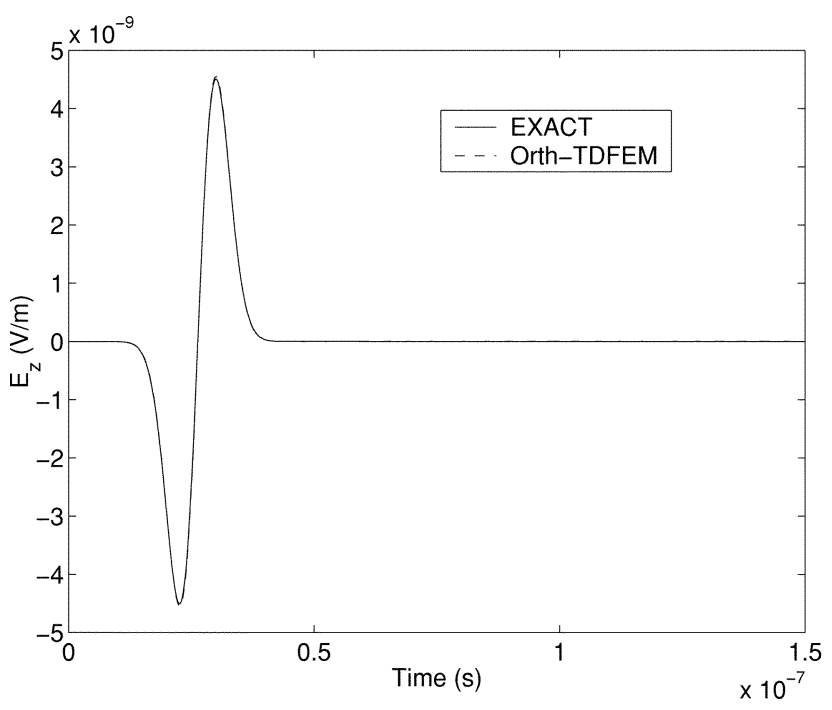

(a)

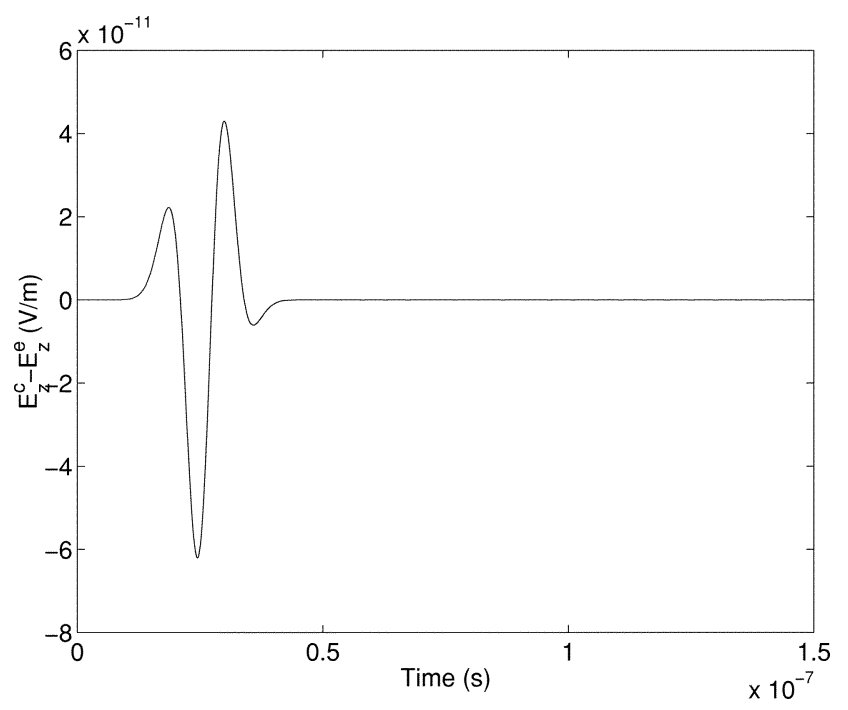

(b)

Fig. 2. Scattering from an empty box of length $1 \mathrm{~m}$, width $0.5 \mathrm{~m}$, and height $0.75 \mathrm{~m}$. (a) Electric field observed at $\mathbf{r}=0.17 \hat{\mathbf{x}}+0.4 \hat{\mathbf{y}}+0.16 \hat{\mathbf{z}} \mathrm{m}$. (b) Absolute error.

\section{ORTHOGONAL TDFEM SOLUTION}

Consider the problem of modeling the electric field $\mathbf{E}$ generated by an external source in a volume $V_{o}$ enclosed by a surface $S_{o}$. Inside $S_{o}$, the electric field $\mathbf{E}$ satisfies

$$
\nabla \times \mu_{r}^{-1} \nabla \times \mathbf{E}(\mathbf{r}, t)+\mu_{0} \epsilon \partial_{t}^{2} \mathbf{E}(\mathbf{r}, t)=0
$$

subject to a mixed boundary condition on $S_{o}$

$$
\mu_{r}^{-1} \hat{n} \times[\nabla \times \mathbf{E}(\mathbf{r}, t)]+c^{-1} \partial_{t} \hat{n} \times[\hat{n} \times \mathbf{E}(\mathbf{r}, t)]=\mathbf{U}(\mathbf{r}, t)
$$

where $\hat{n}$ represents the outward unit vector normal to $S_{o}$.

In accordance with the variational principle [22], the solution to the boundary-value problem defined by (9) and (10) is obtained by seeking the stationary point of the functional (see (11) at the bottom of the page).

Expanding the electric field within each element using the proposed 3-D orthogonal vector bases, we have

$$
\mathbf{E}(\mathbf{r}, t)=\epsilon_{r}^{-1} \sum_{j=1}^{4}\left[b_{j}(t) \mathbf{P}_{j}^{e}(\mathbf{r})+h_{j}(t) \mathbf{Z}_{j}^{e}(\mathbf{r})+t_{j}(t) \mathbf{T}_{j}^{e}(\mathbf{r})\right]
$$

where $b_{j}(t), h_{j}(t)$, and $t_{j}(t)$ denote the corresponding expansion coefficients. Obviously, the above expansion preserves the tangential continuity of the fields across element interfaces (at the central points), whereas allows for the correct normal discontinuity. By substituting (12) into (11), we obtain the ordinary differential equation

$$
\mathbf{M} \frac{d^{2} u}{d t^{2}}+\mathbf{R} \frac{d u}{d t}+\mathbf{S} u+w=0
$$

where $\mathbf{M}, \mathbf{R}$, and $\mathbf{S}$ are square matrices, assembled from their corresponding element matrices given by (14) and (15) at the bottom of the next page and

$$
\mathbf{S}^{e}=\mu_{r}^{-1}\left[\left[\left\langle\nabla \times \epsilon_{r}^{-1} \mathbf{N}_{i}^{e}, \nabla \times \epsilon_{r}^{-1} \mathbf{N}_{j}^{e}\right\rangle\right]\right]
$$

in which $\mathbf{N}_{i}^{e}$ denotes $\mathbf{P}_{i}^{e}, \mathbf{Z}_{i}^{e}$, and $\mathbf{T}_{i}^{e},\langle\cdot, \cdot\rangle$ and $\langle\cdot, \cdot\rangle_{S_{o}}$ denote volume and surface integration, respectively. In (13), $u$ is the unknown vector given by $u=\left[\left\{b_{i}\right\},\left\{h_{i}\right\},\left\{t_{i}\right\}\right]^{T}$, and $w$ is a vector contributed by the excitation on $S_{o}$, which can be assembled from $w^{e}=\left[\left\langle\epsilon_{r}^{-1} \mathbf{P}_{i}^{e}, \mathbf{U}\right\rangle_{S_{o}},\left\langle\epsilon_{r}^{-1} \mathbf{Z}_{i}^{e}, \mathbf{U}\right\rangle_{S_{o}},\left\langle\epsilon_{r}^{-1} \mathbf{T}_{i}^{e}, \mathbf{U}\right\rangle_{S_{o}}\right]^{T}$.

$$
\begin{aligned}
F[\mathbf{E}(\mathbf{r}, t)]= & \frac{1}{2} \iiint_{V_{o}}\left\{\mu_{r}^{-1}[\nabla \times \mathbf{E}(\mathbf{r}, t)] \cdot[\nabla \times \mathbf{E}(\mathbf{r}, t)]+\mu_{0} \epsilon \partial_{t}^{2} \mathbf{E}(\mathbf{r}, t) \cdot \mathbf{E}(\mathbf{r}, t)\right\} d v \\
& +\frac{1}{2} \iint_{S_{o}}\left\{c^{-1} \partial_{t}[\hat{n} \times \mathbf{E}(\mathbf{r}, t)] \cdot[\hat{n} \times \mathbf{E}(\mathbf{r}, t)]+2 \mathbf{E}(\mathbf{r}, t) \cdot \mathbf{U}\right\} d s .
\end{aligned}
$$




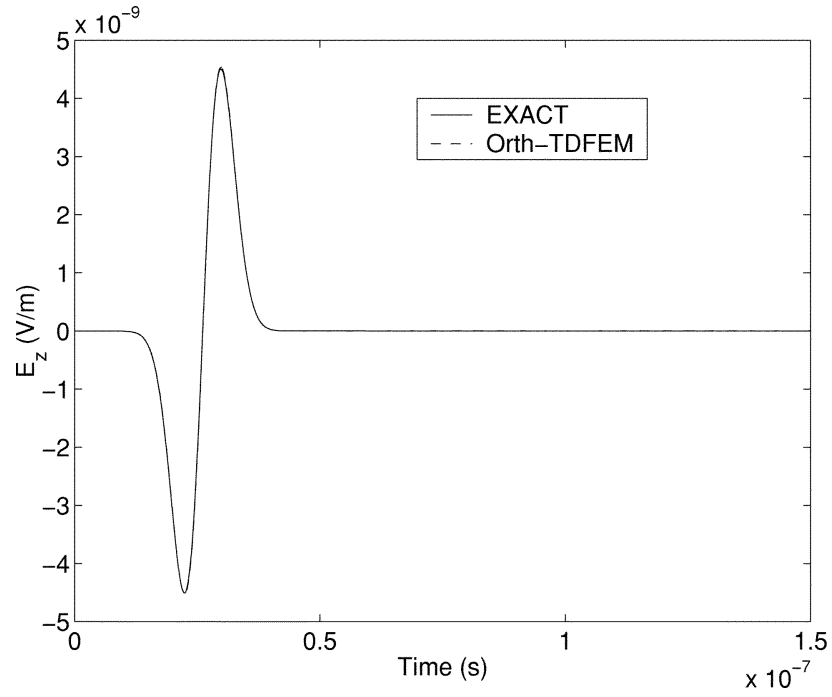

(a)

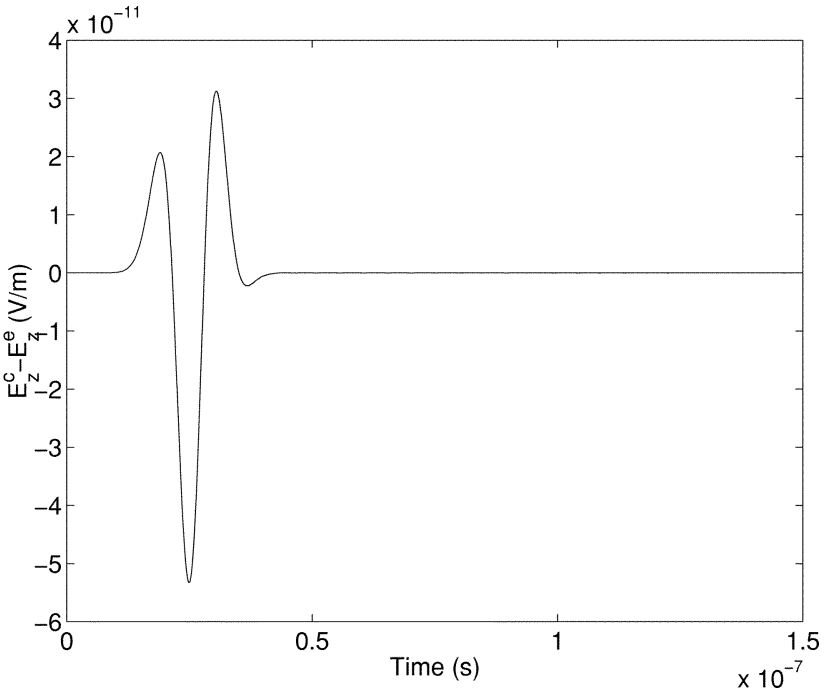

(b)

Fig. 3. Scattering from an empty box of length $1 \mathrm{~m}$, width $0.5 \mathrm{~m}$, and height $0.75 \mathrm{~m}$. (a) Electric field observed at $\mathbf{r}=0.4 \hat{\mathbf{x}}+0.43 \hat{\mathbf{y}}+0.68 \hat{\mathbf{z}} \mathrm{m}$. (b) Absolute error.

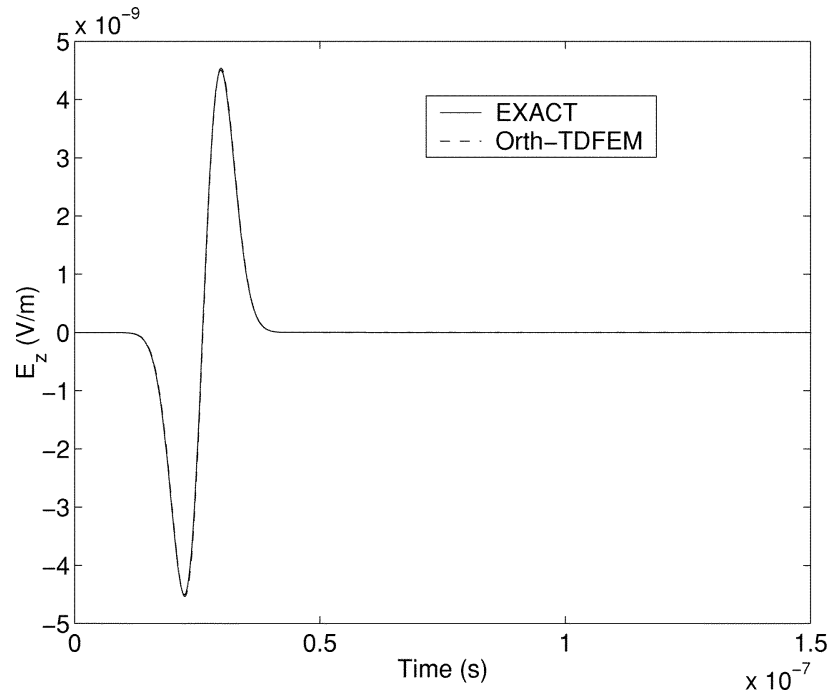

(a)

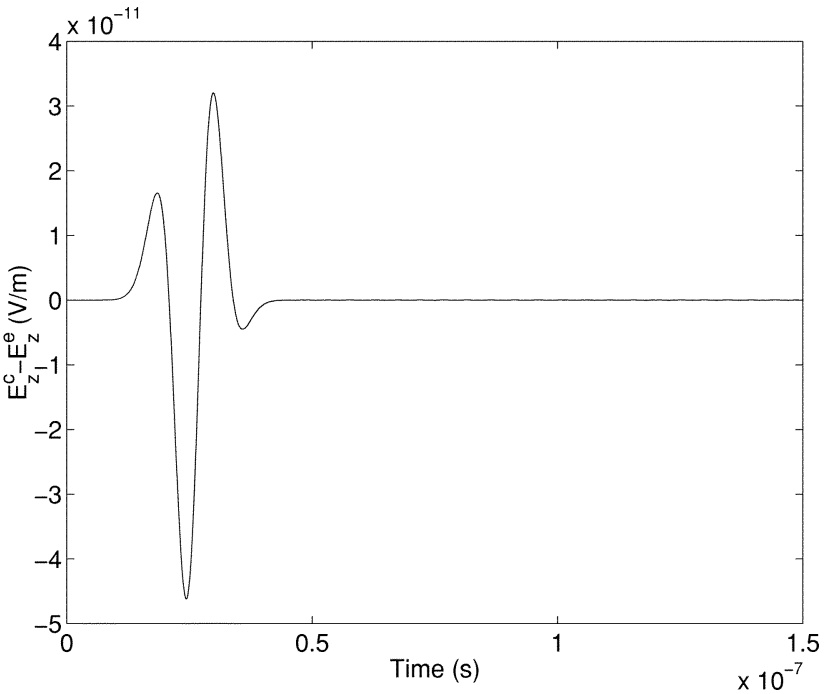

(b)

Fig. 4. Scattering from an empty box of length $1 \mathrm{~m}$, width $0.5 \mathrm{~m}$, and height $0.75 \mathrm{~m}$. (a) Electric field observed at $\mathbf{r}=0.87 \hat{\mathbf{x}}+0.47 \hat{\mathbf{y}}+0.53 \hat{\mathbf{z}} \mathrm{m}$. (b) Absolute error.

Since the expansion functions $\mathbf{P}, \mathbf{Z}$, and $\mathbf{T}$ are orthogonal with each other, matrices $\mathbf{M}$ and $\mathbf{R}$ become diagonal. By adopting central difference to discretize (13) in the axis of time, the matrix solution in every time step is avoided, yielding a purely explicit scheme.

The computational cost of solving (13) scales as $\mathcal{O}\left(N_{t} N\right)$, which is used on a sparse matrix-vector multiplication at each time step, where $N_{t}$ denotes the number of time steps and $N$ the number of unknowns. The efficiency of the orthogonal TDFEM is comparable to that of the FDTD method. Compared to the implicit TDFEM schemes using iterative solvers [12]-[14], [19], the orthogonal TDFEM scheme is more efficient, since the computational cost of these implicit schemes scales as $\mathcal{O}\left(N_{t} N_{i t} N\right)$ [12], where $N_{i t}$ stands for the number of iterations per time step.

$$
\begin{aligned}
& \mathbf{M}^{e}=\mu_{0} \epsilon_{0}\left[\begin{array}{lll}
{\left[\left\langle\epsilon_{r}^{-1} \mathbf{P}_{i}^{e}, \epsilon_{r}^{-1} \mathbf{P}_{j}^{e}\right\rangle\right]} & & \\
& {\left[\left\langle\epsilon_{r}^{-1} \mathbf{Z}_{i}^{e}, \epsilon_{r}^{-1} \mathbf{Z}_{j}^{e}\right\rangle\right]} & \\
& {\left[\left\langle\epsilon_{r}^{-1} \mathbf{T}_{i}^{e}, \epsilon_{r}^{-1} \mathbf{T}_{j}^{e}\right\rangle\right]}
\end{array}\right] \\
& \mathbf{R}^{e}=c^{-1}\left[\begin{array}{lll}
{[0]} & \left.\left[\hat{n} \times \epsilon_{r}^{-1} \mathbf{Z}_{i}^{e}, \hat{n} \times \epsilon_{r}{ }^{-1} \mathbf{Z}_{j}^{e}\right\rangle_{S_{o}}\right] & \\
& {\left[\left\langle\hat{n} \times \epsilon_{r}^{-1} \mathbf{T}_{i}^{e}, \hat{n} \times \epsilon_{r}{ }^{-1} \mathbf{T}_{j}^{e}\right\rangle_{S_{o}}\right]}
\end{array}\right]
\end{aligned}
$$




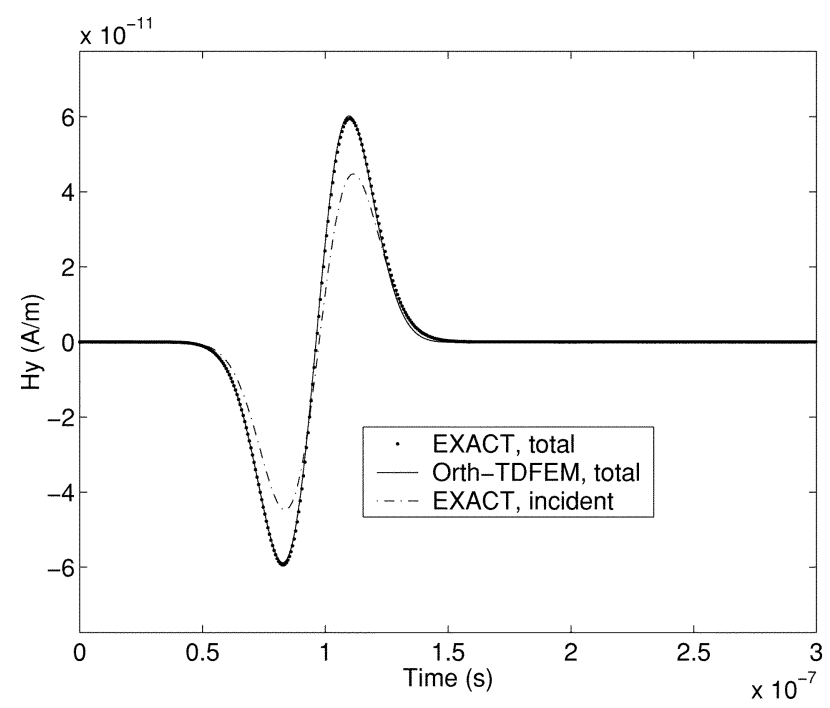

(a)

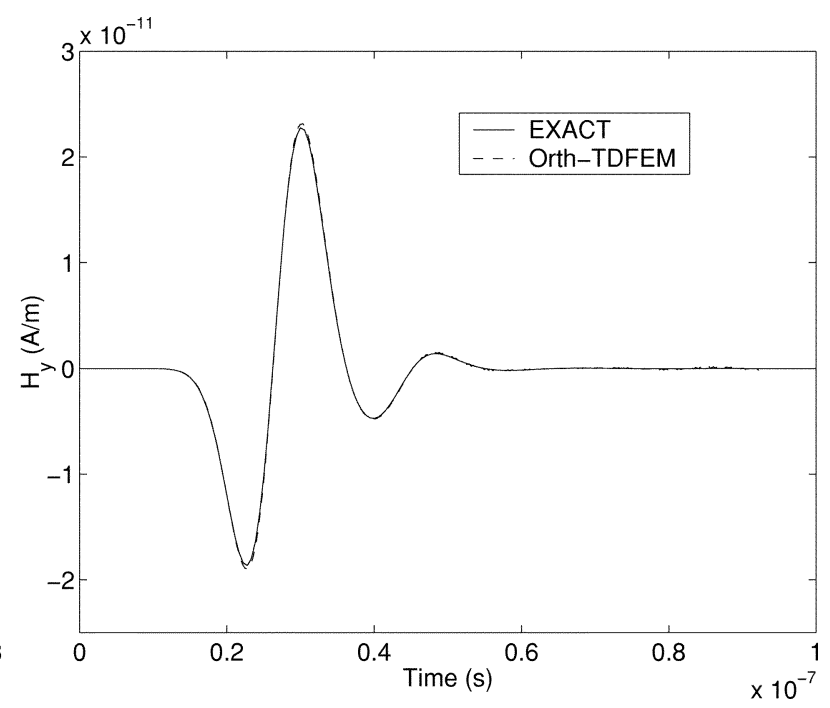

(b)

Fig. 5. Scattering from a perfect conducting sphere of radius $0.8 \mathrm{~m}$. (a) Magnetic field observed at $\mathbf{r}=-0.01 \hat{\mathbf{x}}+0.035 \hat{\mathbf{y}}-0.96 \hat{\mathbf{z}} \mathrm{m}$ with incident pulse parameter $t_{0}=165 \mathrm{~ns}$ and $\tau=33.3 \mathrm{~ns}$. (b) Magnetic field observed at $\mathbf{r}=-0.01 \hat{\mathbf{x}}+0.035 \hat{\mathbf{y}}-0.96 \hat{\mathbf{z}} \mathrm{m}$ with incident pulse parameter $t_{0}=25.99 \mathrm{~ns}$ and $\tau=5.25 \mathrm{~ns}$

Many factors affect the number of $N_{i t}$ such as the property of the matrix to be solved, the length of the time step, the choice of the iterative solver together with the corresponding preconditioner, and the required accuracy. For a 3-D FEM matrix system constructed from the zeroth-order vector basis functions (edge elements), if the conjugate gradient (CG) method with diagonal preconditioning is used, $N_{i t}$ is usually required to be 10-20 to reach a reasonable accuracy [14], [19]. For a 3-D FEM matrix system constructed from higher-order vector basis functions [23], the required $N_{i t}$ increases drastically due to the deterioration of the matrix condition. The reduction of $N_{i t}$ relies on the construction of robust preconditioners, which has yet to be fully exploited. On the other hand, although implicit schemes can be formulated to be unconditionally stable [12], [14], [19], the time step cannot be chosen arbitrarily large; therefore, $N_{t}$ cannot be chosen arbitrarily small. This is because the time step is upper bounded by Shannon's sampling limit so that the spectral content of the temporal signal can be accommodated, and although the time step is not constrained by the stability criterion, it is limited by the accuracy requirement. It is observed that in order to balance both accuracy and efficiency, the time step can only be chosen a little bit larger than that required by conditionally stable schemes such as the central differencing scheme [14].

\section{STABILITY ANALYSIS}

In accordance with the general approach derived in [24], we deduce that the stability of the orthogonal TDFEM can be ensured as long as the time step satisfies

$$
\Delta t \leq \frac{2}{\sqrt{\rho\left(\mathbf{M}^{-1} \mathbf{S}\right)}}
$$

in which $\rho(\cdot)$ denotes the spectral radius of matrix $(\cdot)$. Based on the construction rules of the 3 -D orthogonal bases, it is clear that the mass matrix $\mathbf{M}$ is preserved to be positive definite and the matrix $\mathbf{S}$ is kept semipositive definite. Therefore, the stability of the TDFEM scheme based on the proposed 3-D orthogonal vector bases is always guaranteed.

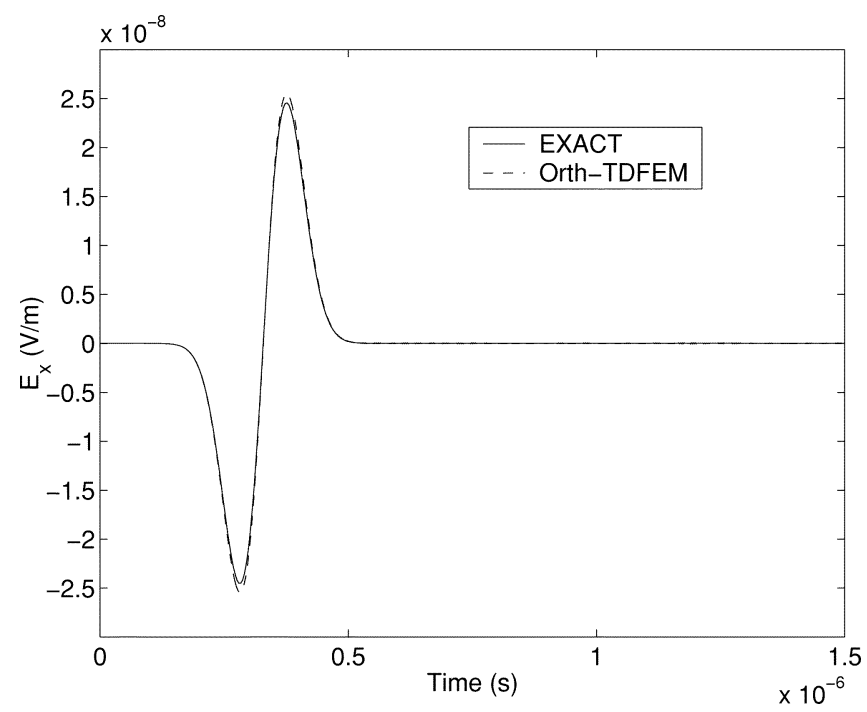

Fig. 6. Scattering from a dielectric coated sphere of radius $0.8 \mathrm{~m}$. The dielectric coating has a thickness of $0.2 \mathrm{~m}$ and a relative permittivity of $\epsilon_{r}=4.0$

An example investigated here is an empty box of length $1 \mathrm{~m}$, width $0.5 \mathrm{~m}$, and height $0.75 \mathrm{~m}$. The computational domain is discretized into 40 tetrahedra, resulting in 300 unknowns. By an eigenvalue analysis of matrix $\mathbf{M}^{-1} \mathbf{S}$, it is revealed that the spectral radius is equal to $2.91 \times 10^{20}$. Hence from (17), to guarantee stability, the time step should be less than $0.12 \mathrm{~ns}$. This agrees with the result from our numerical experiments.

\section{NUMERICAL EXAMPLES}

To validate the proposed 3-D orthogonal vector basis functions, we first consider the scattering from an empty box (a dielectric volume with $\epsilon_{r}=\mu_{r}=1$ ) as used in the preceding section on stability analysis. The box is discretized into 494 tetrahedra generating 2882 unknowns. The parameters defining the incident Neumann pulse [20], [21] are $\hat{\mathbf{k}}=-\hat{\mathbf{y}}, \hat{\mathbf{E}}=\hat{\mathbf{z}}$, $\mathbf{r}_{0}=0.5 \hat{\mathbf{x}}+0.5 \hat{\mathbf{y}}+0.375 \hat{\mathbf{z}} \mathrm{m}, t_{0}=25.99 \mathrm{~ns}$, and $\tau=$ 


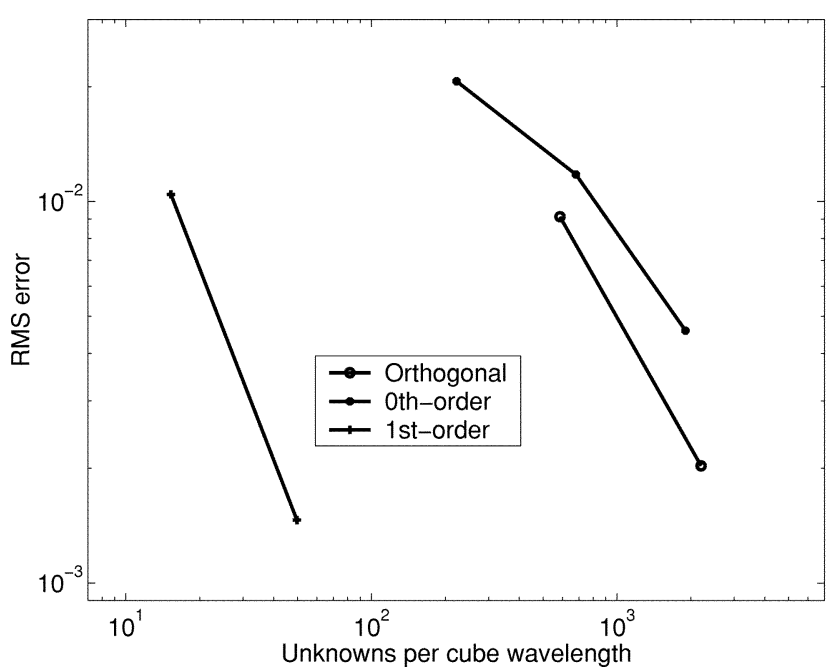

(a)

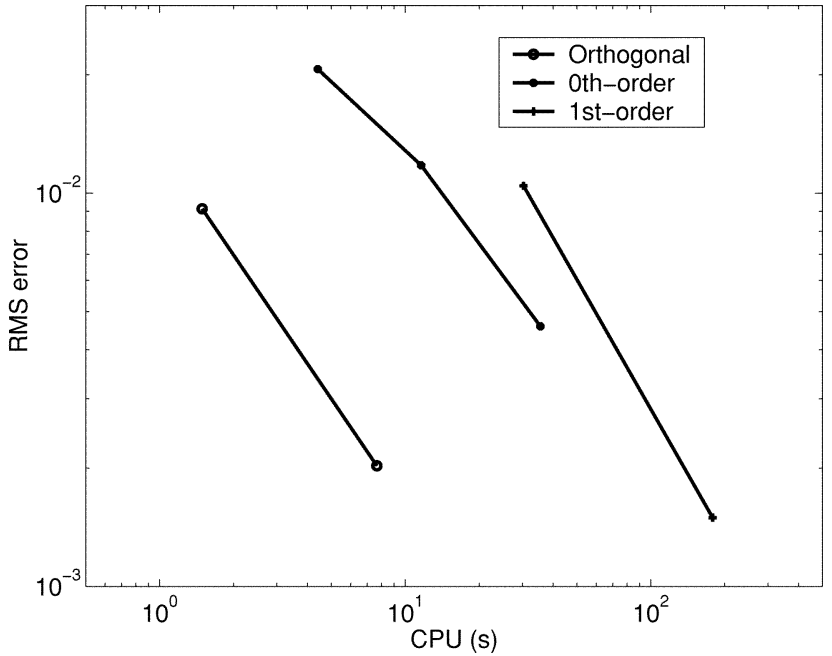

(b)

Fig. 7. (a) RMS error versus the number of unknowns per cube wavelength. (b) RMS error versus CPU time.

$5.25 \mathrm{~ns}$. The $z$-component of the electric field observed at $\mathbf{r}=$ $0.17 \hat{\mathbf{x}}+0.4 \hat{\mathbf{y}}+0.16 \hat{\mathbf{z}} \mathrm{m}, \mathbf{r}=0.4 \hat{\mathbf{x}}+0.43 \hat{\mathbf{y}}+0.68 \hat{\mathbf{z}} \mathrm{m}$, and $\mathbf{r}=0.87 \hat{\mathbf{x}}+0.47 \hat{\mathbf{y}}+0.53 \hat{\mathbf{z}} \mathrm{m}$ together with the absolute error are shown in Figs. 2-4, respectively. Excellent agreement is observed between the calculated and exact results.

Next, we consider the scattering from a perfect conducting sphere of radius $0.8 \mathrm{~m}$. The computational domain is discretized into 4307 tetrahedra, yielding 27885 unknowns. An $\hat{\mathbf{x}}$ polarized Neumann pulse is normally incident upon the sphere along the $\hat{\mathbf{z}}$ direction. The incident pulse parameters are $\mathbf{r}_{0}=-\hat{\mathbf{z}} \mathrm{m}$, $t_{0}=165 \mathrm{~ns}$, and $\tau=33.3 \mathrm{~ns}$. Fig. 5(a) shows the incident as well as the calculated total magnetic field at $\mathbf{r}=-0.01 \hat{\mathbf{x}}+$ $0.035 \hat{\mathbf{y}}-0.96 \hat{\mathbf{z}} \mathrm{m}$, which agrees very well with the exact result. Fig. 5(b) shows the calculated magnetic field at the same observation point except that the incident pulse parameters are changed to $t_{0}=25.99 \mathrm{~ns}$ and $\tau=5.25 \mathrm{~ns}$. Again, the calculated field is in excellent agreement with the exact data.

To examine the capability of the proposed basis functions to model the fields in an inhomogeneous environment, we consider a dielectric coated sphere of radius $0.8 \mathrm{~m}$. The dielectric coating has a thickness of $0.2 \mathrm{~m}$ and a relative permittivity of $\epsilon_{r}=4.0$. The computational region is subdivided into 1956 tetrahedra, generating 12549 unknowns. The incident pulse parameters are $\hat{\mathbf{k}}=\hat{\mathbf{z}}, \hat{\mathbf{E}}=\hat{\mathbf{x}}, \mathbf{r}_{0}=1.2 \hat{\mathbf{x}}+1.2 \hat{\mathbf{y}} \mathrm{m}, t_{0}=0.33 \mu \mathrm{s}$, and $\tau=0.067 \mu$ s. The calculated $x$-component of the electric field at $\mathbf{r}=1.16 \hat{\mathbf{x}}+1.44 \hat{\mathbf{y}}+0.1 \hat{\mathbf{z}} \mathrm{m}$ is displayed in Fig. 6 together with the exact solution.

Finally, we examine the accuracy and efficiency of the proposed 3-D orthogonal vector bases in comparison with the traditional zeroth- and first-order vector bases. The numerical experiments are performed on the empty box as depicted in the first example. The incident pulse is characterized by the same set of parameters. Three meshes are generated which subdivide the box into 40, 146, and 494 tetrahedra, respectively. For each mesh, calculations are carried out using the orthogonal, the zeroth-, and the first-order vector basis functions. The CG solver with diagonal preconditioning is employed to solve the matrix in each time step for the calculations using the zeroth- or first-order vector bases. The central differencing scheme is used to carry

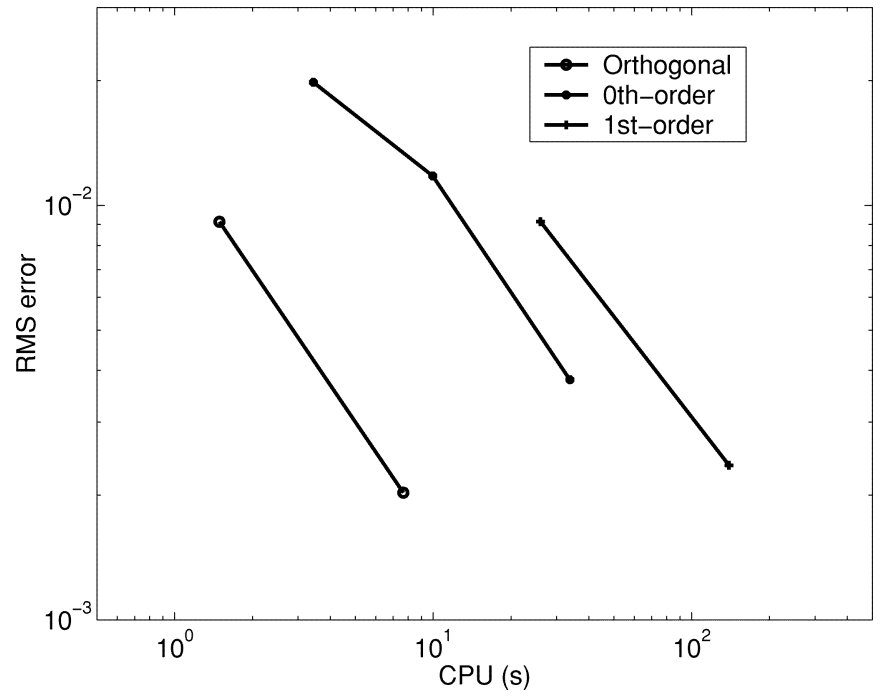

Fig. 8. RMS error versus CPU time with the zeroth- and the first-order results generated by uncontionally stable TDFEM schemes.

out the time discretization. The root mean square (rms) error, normalized by the maximum amplitude, in the $x$-component of the magnetic field observed at $\mathbf{r}=0.35 \hat{\mathbf{x}}+0.41 \hat{\mathbf{y}}+0.1 \hat{\mathbf{z}} \mathrm{m}$ is plotted as a function of the unknown density in Fig. 7(a). The RMS error with respect to the computing time is plotted in Fig. 7(b). It is evident that to achieve a required accuracy, the TDFEM that adopts 3-D orthogonal vector bases is more efficient. Next, to compare with the unconditionally stable implicit TDFEM schemes, the Newmark method with parameter $\gamma=0.5, \beta=0.25$ [12], [14], [19] is utilized to perform the time discretization for the calculation based on the zeroth- and the first-order vector basis functions. Fig. 8 shows the calculated RMS error versus the CPU time. Once again, the superior efficiency of the TDFEM scheme using the proposed 3-D orthogonal vector bases is demonstrated clearly. (Note that the first-order TDFEM can be made faster using more advanced direct solvers such as the multifrontal algorithm used in [21] or iterative solvers with a better preconditioner, which has not been fully studied in TDFEM.) 


\section{CONCLUSION}

This paper presents 3-D orthogonal vector basis functions for the TDFEM solution of vector wave equations. The use of the proposed basis functions completely eliminates the matrix solution at each time step. Defined over tetrahedral elements, these basis functions permit the accurate modeling of complex geometry. In contrast to the traditional vector bases, these orthogonal bases enforce both the tangential continuity of the field and the normal continuity of the flux explicitly at the interpolation points. The stability of the resulting TDFEM scheme is shown to be ensured. The accuracy and efficiency of these basis functions are demonstrated by numerical results together with the comparison with the traditional vector basis functions. It is hoped that the proposed 3-D orthogonal vector basis functions will contribute to the future popularity of the time-domain finite element method.
[17] J. R. Brauer, R. Mittra, and J. F. Lee, "Absorbing boundary condition for vector and scalar potentials arising in electromagnetic finite element analysis in frequency and time domains," IEEE APS Int. Symp. Dig., pp. 1224-1227, 1991.

[18] K. Mahadevan, R. Mittra, D. Rowse, and J. Murphy, "Edge-based finite element frequency and time domain algorithms for RCS computation," IEEE APS Int. Symp. Dig., vol. 3, pp. 1680-1683, 1993.

[19] W. P. Carpes Jr, L. Pichon, and A. Razek, "A 3D finite element method for the modeling of bounded and unbounded electromagnetic problems in the time domain," Int. J. Numer. Model., vol. 13, pp. 527-540, 2000.

[20] D. Jiao, M. Lu, E. Michielssen, and J. M. Jin, "A fast time-domain finite element-boundary integral method for electromagnetic analysis," IEEE Trans. Antennas Propagat., vol. 49, pp. 1453-1461, Oct. 2001.

[21] D. Jiao, A. A. Ergin, B. Shanker, E. Michielssen, and J. M. Jin, "A fast higher-order time-domain finite element-boundary integral method for 3-D electromagnetic scattering analysis," IEEE Trans. Antennas Propagat., vol. 50, pp. 1192-1202, Sept. 2001.

[22] J. M. Jin, The Finite Element Method in Electromagnetics, New York: Wiley, 1993.

[23] R. D. Graglia, D. R. Wilton, and A. F. Peterson, "Higher order interpolatory vector bases for computational electromagnetics," IEEE Trans. Antennas Propagat., vol. 45, pp. 329-341, Mar. 1997.

[24] D. Jiao and J. M. Jin, "A general approach for the stability analysis of time-domain finite element method," IEEE APS Int. Symp. Dig., 2001.

\section{REFERENCES}

[1] K. S. Yee, "Numerical solution of initial boundary value problems involving Maxwell's equations in isotropic media," IEEE Trans. Antennas Propagat., vol. 14, pp. 302-307, May 1966.

[2] J. F. Lee, R. Lee, and A. C. Cangellaris, "Time-domain finite element methods," IEEE Trans. Antennas Propagat., vol. 45, pp. 430-442, Mar. 1997.

[3] C. L. Bennet, "A Technique for Computing Approximate Impulse Response for Conducting Bodies," Ph.D., Purdue University, 1968.

[4] R. Mittra, "Integral equation methods for transient scattering," in Transient Electromagnetic Fields, L. B. Felsen, Ed. New York: Springer Verlag, 1976, ch. 2.

[5] S. M. Rao and D. R. Wilton, "Transient scattering by conducting surfaces of arbitrary shape," IEEE Trans. Antennas Propagat., vol. 39, pp. 56-61, Jan. 1991.

[6] A. C. Cangellaris, C. C. Lin, and K. K. Mei, "Point-matched time-domain finite element methods for electromagnetic radiation and scattering," IEEE Trans. Antennas Propagat., vol. 35, pp. 1160-1173, Oct. 1987

[7] J. T. Elson, H. Sangani, and C. H. Chan, "An explicit time-domain method using three-dimensional Whitney elements," Microwave Opt. Technol. Lett., vol. 7, pp. 607-610, Sept. 1994.

[8] M. F. Wong, O. Picon, and V. F. Hanna, "A finite-element method based on Whitney forms to solve Maxwell equations in the time-domain," IEEE Trans. Magn., vol. 31, pp. 1618-1621, May 1995.

[9] K. Mahadevan and R. Mittra, "Radar cross section computation of inhomogeneous scatterers using edge-based finite element methods in frequency and time domains," Radio Sci., vol. 28, pp. 1181-1193, Nov.-Dec. 1993.

[10] D. R. Lynch and K. D. Paulsen, "Time-domain integration of the Maxwell equations on finite elements," IEEE Trans. Antennas Propagat., vol. 38, pp. 1933-1942, Mar. 1991.

[11] G. Mur, "The finite-element modeling of three-dimensional time-domain electromagnetic fields in strongly inhomogeneous media," IEEE Trans. Magn., vol. 28, pp. 1130-1133, Mar. 1992.

[12] J. F. Lee and Z. Sacks, "Whitney elements time domain (WETD) methods," IEEE Trans. Magn., vol. 31, pp. 1325-1329, May 1995.

[13] J. F. Lee, "WETD-a finite-element time-domain approach for solving Maxwell's equations," IEEE Microwave Guided Wave Lett., vol. 4, pp. 11-13, Jan. 1994.

[14] S. D. Gedney and U. Navsariwala, "An unconditionally stable finite-element time-domain solution of the vector wave equation," IEEE Microwave Guided Wave Lett., vol. 5, pp. 332-334, May 1995.

[15] D. A. White, "Orthogonal vector basis functions for time domain finite element solution of the vector wave equation," IEEE Trans. Magn., vol. 35, pp. 1458-1461, May 1999.

[16] J. M. Jin, M. Zunoubi, K. C. Donepudi, and W. C. Chew, "Frequencydomain and time-domain finite-element solution of Maxwell's equations using spectral Lanczos decomposition method," Comput. Methods Appl. Mech. Engrg., vol. 169, pp. 279-296, 1999.

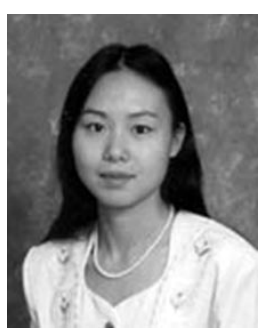

Dan Jiao (S'00-M'02) was born in Anhui Province, China, in 1972. She received the B.S. and M.S. degrees in electrical engineering from Anhui University, China, in 1993 and 1996, respectively, and the Ph.D. degree in electrical engineering from the University of Illinois at Urbana-Champaign in 2001.

From 1996 to 1998 , she performed graduate studies at the University of Science and Technology of China, Hefei, China. From 1998 to 2001, she was a Research Assistant at the Center for Computational Electromagnetics, University of Illinois at Urbana-Champaign. She was the recipient of the 2000 Raj Mittra Outstanding Research Award presented by the Department of Electrical and Computer Engineering, University of Illinois at Urbana-Champaign. In 2001, she joined Intel Corporation, Santa Clara, CA. Her current research interests include fast computational methods in electromagnetics and time-domain numerical techniques.

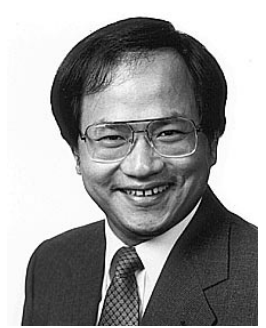

Jian-Ming Jin (S'87-M'89-SM'94-F'01) received the B.S. and M.S. degrees in applied physics from Nanjing University, Nanjing, China, in 1982 and 1984, respectively, and the Ph.D. degree in electrical engineering from the University of Michigan, Ann Arbor, in 1989.

He is a Full Professor of Electrical and Computer Engineering and Associate Director of the Center for Computational Electromagnetics at the University of Illinois at Urbana-Champaign. He has authored or coauthored more than 120 papers in refereed journals and 15 book chapters. He has also authored The Finite Element Method in Electromagnetics (New York: Wiley, 1993) and Electromagnetic Analysis and Design in Magnetic Resonance Imaging (Boca Raton, FL: CRC, 1998), coauthored Computation of Special Functions (New York: Wiley, 1996), and coedited Fast and Efficient Algorithms in Computational Electromagnetics (Norwood, MA: Artech, 2001). His current research interests include computational electromagnetics, scattering and antenna analysis, electromagnetic compatibility, and magnetic resonance imaging. His name is often listed in the University of Illinois at Urbana-Champaign's List of Excellent Instructors. He currently serves as an Associate Editor of Radio Science and is also on the Editorial Board for Electromagnetics Journal and Microwave and Optical Technology Letters. 
Dr. Jin is a member of Commision B of USNC/URSI, Tau Beta Pi, and International Society for Magnetic Resonance in Medicine. He was a recipient of the 1994 National Science Foundation Young Investigator Award and the 1995 Office of Naval Research Young Investigator Award. He also received the 1997 Xerox Junior Research Award and the 2000 Xerox Senior Research Award presented by the College of Engineering, University of Illinois at Urbana-Champaign, and was appointed as the first Henry Magnuski Outstanding Young Scholar in the Department of Electrical and Computer Engineering in 1998. He was a Distinguished Visiting Professor in the Air Force Research Laboratory in 1999. He served as an Associate Editor of the IEEE TRANSACTIONS ON ANTENNAS AND PROPAGATION (1996-1998). He was the Symposium Co-chairman and Technical Program Chairman of the Annual Review of Progress in Applied Computational Electromagnetics in 1997 and 1998, respectively. 